\title{
Successful diagnosis of tuberculous lymphadenitis by loop-mediated isothermal amplification of cutaneous samples from an ulcerated surface lesion: a case report
}

\author{
Shuichi Kawano1, Takuya Maeda1*, Junichi Watanabe², Yuji Fujikura', Kei Mikita', Yu Hara', Soichiro Kanoh', \\ Fumihiko Kimura ${ }^{2}$, Yasushi Miyahira ${ }^{3}$ and Akihiko Kawana ${ }^{1}$
}

\begin{abstract}
Introduction: Tuberculous lymphadenitis is the most frequent form of extrapulmonary tuberculous. Although nucleic acid amplification assays such as polymerase chain reaction have recently become mainstream techniques for diagnosing tuberculous lymphadenitis, they are still not routinely performed in developing countries because of their high costs and complicated procedures.

Case presentation: We describe a case of tuberculous lymphadenitis in a 79-year-old Japanese man who had been on continuous hemodialysis for end-stage renal disease. We employed loop-mediated isothermal amplification and the procedure for ultrarapid extraction to develop a fast and easy-to-perform procedure for diagnosing tuberculous lymphadenitis.
\end{abstract}

Conclusions: The commercially available loop-mediated isothermal amplification assay kit and a rapid purification procedure enabled us to identify and amplify a Mycobacterium tuberculosis-specific gene within just 1.5 hours.

Keywords: Fine-needle aspiration, Loop-mediated isothermal amplification, Mycobacterium tuberculosis, Tuberculous lymphadenitis

\section{Introduction}

Tuberculous (TB) lymphadenitis is the most frequent form of extrapulmonary tuberculosis and cervical lymphadenopathy is its most common clinical manifestation [1]. Because lymphadenopathy can be attributed to multiple etiologies, such as malignancy, autoimmune disease and infection, it is often difficult to differentiate TB lymphadenitis from other forms of lymphadenitis on clinical grounds alone. The advent of novel diagnostic techniques, in particular loop-mediated isothermal amplification (LAMP), provides accessible, cost-effective, easy-to-perform methods for diagnosing pulmonary TB by examination of respiratory specimens [2]. Boehme et al. demonstrated that LAMP has a diagnostic success rate of $97.7 \%$ for smear-positive

\footnotetext{
* Correspondence: tmaeda@ndmc.ac.jp

'Division of Infectious Diseases and Pulmonary Medicine, Department of Internal Medicine, National Defense Medical College, 3-2, Namiki, Tokorozawa, Saitama 359-8513, Japan

Full list of author information is available at the end of the article
}

sputum specimens and $48.8 \%$ for smear-negative but culture-positive specimens [3]. In our present case, to detect Mycobacterium tuberculosis in a patient diagnosed with TB lymphadenitis, we examined boiled swab samples taken from an ulcerative lesion by LAMP after applying the procedure for ultrarapid extraction (PURE) to isolate target deoxyribonucleic acid (DNA) molecules for use as LAMP reagents.

\section{Case presentation}

A 79-year-old Japanese man who had been on continuous hemodialysis for end-stage renal disease since 2004 visited an outpatient clinic complaining of a unilateral cervical mass and intermittent fever of more than three months' duration. An examination at the clinic revealed three palpable right cervical lymph nodes. His body temperature was $37.4^{\circ} \mathrm{C}$, with no chills or night sweats. Key laboratory data upon admission are shown in Table 1. 
Table 1 Laboratory findings upon admission ${ }^{a}$

\begin{tabular}{|c|c|c|c|}
\hline \multicolumn{2}{|c|}{ Peripheral blood } & \multicolumn{2}{|c|}{ Blood chemistry } \\
\hline WBCs & $6800 / \mu \mathrm{L}$ & T-bil & $0.3 \mathrm{mg} / \mathrm{dL}$ \\
\hline RBCs & $439 \times 10^{4} / \mu \mathrm{L}$ & AST & $14 I U / L$ \\
\hline $\mathrm{Hb}$ & $11.4 \mathrm{~g} / \mathrm{dL}$ & ALT & 7IU/L \\
\hline $\mathrm{Hct}$ & $37.2 \%$ & $\mathrm{LDH}$ & 190IU/L \\
\hline PLT & $23.0 \times 10^{4} / \mu \mathrm{L}$ & ALP & $3211 \mathrm{U} / \mathrm{L}$ \\
\hline Seg & $65.3 \%$ & Y-GTP & $23 I U / L$ \\
\hline Eos & $2.1 \%$ & TP & $6.4 \mathrm{~g} / \mathrm{dL}$ \\
\hline Ly & $19.3 \%$ & BUN & $20.0 \mathrm{mg} / \mathrm{dL}$ \\
\hline \multirow[t]{9}{*}{ Mo } & $12.9 \%$ & $\mathrm{Cr}$ & $5.65 \mathrm{mg} / \mathrm{dL}$ \\
\hline & & CPK & 19.0IU/L \\
\hline & & $\mathrm{Na}^{+}$ & $140 \mathrm{mEq} / \mathrm{L}$ \\
\hline & & $\mathrm{K}^{+}$ & $3.9 \mathrm{mEq} / \mathrm{L}$ \\
\hline & & $\mathrm{Cl}$ & $101 \mathrm{mEq} / \mathrm{L}$ \\
\hline & & Glc & $88 \mathrm{mg} / \mathrm{dL}$ \\
\hline & & CRP & $<0.05 \mathrm{mg} / \mathrm{dL}$ \\
\hline & & ESR & $74 \mathrm{~mm} /$ hour \\
\hline & & sIL-2R & $2.05 \mathrm{U} / \mathrm{mL}$ \\
\hline
\end{tabular}

${ }^{a}$ ALP, Alkaline phosphatase; ALT, Alanine aminotransferase; AST, Alanine aminotransferase; BUN, Blood urea nitrogen; $\mathrm{Cl}$, Chloride; $\mathrm{CPK}$, Creatine phosphokinase; $\mathrm{Cr}$, Creatinine; CRP, C-reactive protein; Eos, Eosinophils; ESR, Erythrocyte sedimentation rate; Glc, Glucose; $\gamma$-GTP, $\gamma$-Glutamyl transpeptidase; $\mathrm{Hb}$, Hemoglobin; Hct, Hematocrit; $\mathrm{K}^{+}$, Potassium; LDH, Lactate dehydrogenase; Ly, Lymphocytes; Mo, Monocytes; $\mathrm{Na}^{+}$, Sodium; PLT, Platelets; RBC, Red blood cells; Seg, Segmented neutrophils; sIL-2R, Soluble interleukin 2 receptor; T-bil, Total bilirubin; TP, Total protein; WBC, White blood cells.

The findings of the lab test for human immunodeficiency virus (HIV) were negative. Computed tomography of the neck and chest revealed multiple enlarged lymph nodes measuring $40 \mathrm{~mm} \times 20 \mathrm{~mm}$ with low central attenuation predominantly along the lateral margin of the right sternocleidomastoid muscle (Figure 1A). In addition, several enlarged lymph nodes measuring $29 \mathrm{~mm} \times 30 \mathrm{~mm}$ in the supraclavicular region were also noted (Figure 1B). The result of an assay for interferon $\gamma$ release was positive [4].

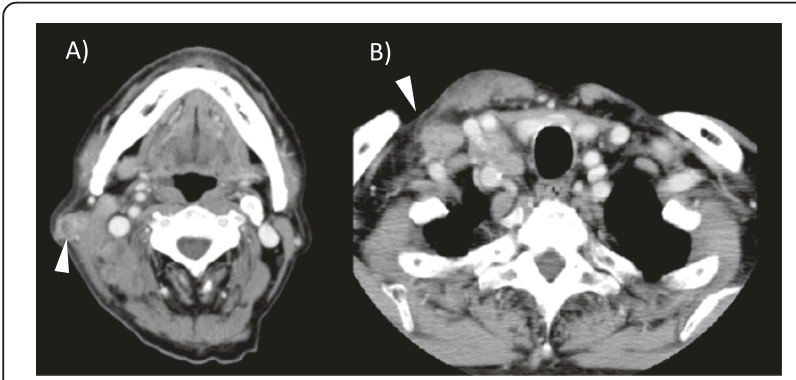

Figure 1 Contrast-enhanced computed tomography scan. Arrowheads point to multiple enlarged lymph nodes at the lateral margin of the right sternocleidomastoid muscle (A) and in the supraclavicular region (B)
Suspecting TB lymphadenitis, we performed fine-needle aspiration (FNA) of the para-sternocleidomastoid nodes. The obtained specimens were examined by microscopy (smears for acid-fast bacilli and fungi), microbiological culture and cytology. The smears were negative for acid-fast bacilli, and the cytology was determined to be class II, partly due to the presence of a mass of neutrophils. Among the diagnostic procedures we used, only the six-week culture for tuberculosis with the BacT/ ALERT $^{\bullet}$ three-dimensional microbial detection system (bioMérieux, Nürtingen, Germany) was positive, and $M$. tuberculosis DNA was unequivocally identified in the positive culture by means of the COBAS $\mathrm{TaqMan}^{\circ} \mathrm{MTB}$ assay (Roche Diagnostics, Rotkreuz, Switzerland) [5].

In this case, we reached a definitive diagnosis of TB lymphadenitis in an efficient manner by using a commercially available LAMP kit (Eiken Chemical, Tokyo, Japan) to amplify an M. tuberculosis-specific gene and a PURE kit (Eiken Chemical) to isolate the DNA target of interest [6]. Cutaneous swab samples were obtained by wiping the surface of an ulcerated lesion (Figure 2) with a sterile cotton swab and placing it into $960 \mu \mathrm{L}$ of DNA extraction solution. The suspension was incubated on a heat block at $100^{\circ} \mathrm{C}$ for 10 minutes, and DNA was rapidly extracted with the PURE kit in $30 \mu \mathrm{L}$ of solution [6]. Amplification of the M. tuberculosis-specific gene was achieved by LAMP at $64^{\circ} \mathrm{C}$ for 40 minutes according to the manufacturer's instructions, and a positive LAMP result was indicated by the color change observed under ultraviolet light (Figure 3). On the basis of these results, we diagnosed TB lymphadenitis.

The patient subsequently received anti-tuberculosis chemotherapy consisting of isonicotinic acid hydrazide $(300 \mathrm{mg} /$ day), rifampicin $(450 \mathrm{mg} /$ day $)$ and ethambutol hydrochloride $(500 \mathrm{mg} /$ day after dialysis). Two weeks later, he became afebrile and had no signs of

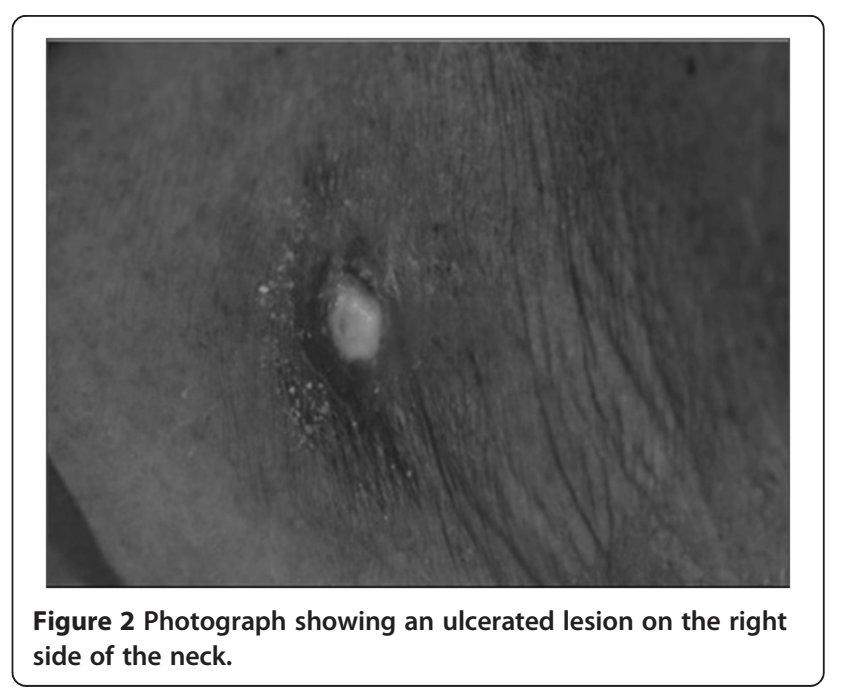




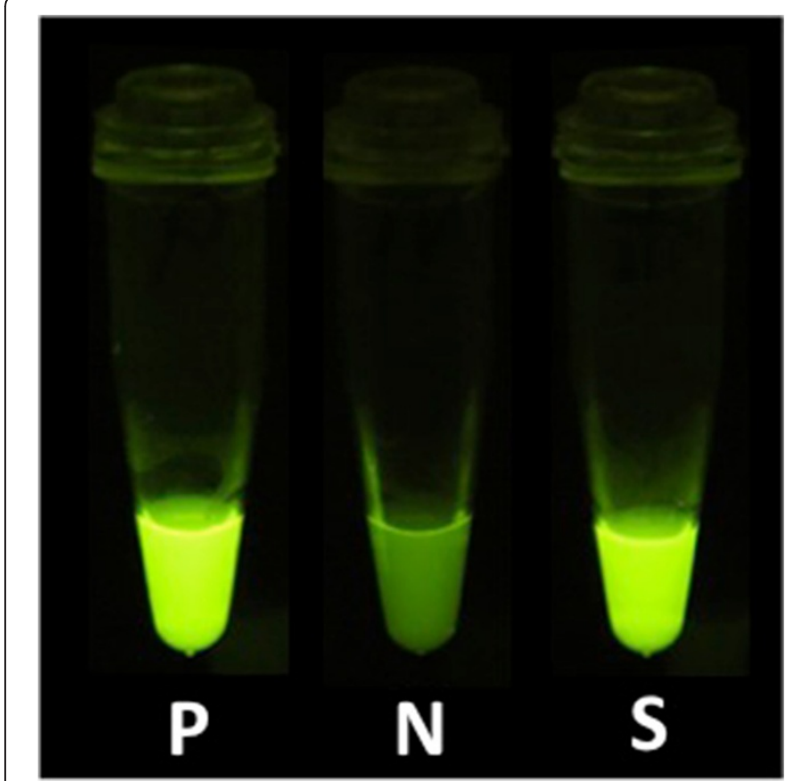

Figure 3 Detection of Mycobacterium tuberculosis by loop-mediated isothermal amplification assay showing the color change to cloudy yellow. N: negative control; P: positive control; S: clinical sample.

exacerbation, and his painful lymphadenopathy eventually subsided.

\section{Discussion}

The current gold standard methods for diagnosing tuberculosis are microscopy and culture. However, the poor sensitivity of microscopy and the laborious, time-consuming procedures of culturing make these modalities less than ideal. Therefore, nucleic acid amplification assays such as polymerase chain reaction (PCR) have recently become mainstream techniques for diagnosing tuberculosis [7]. However, the molecular biological technologies on which such methods are based require not only experience and expertise but also expensive equipment; this is the principal reason why most of these sophisticated diagnostic tools are not suitable for use in resource-limited, disease-endemic regions. In contrast, LAMP can amplify M. tuberculosisspecific DNA rapidly under isothermal conditions [8]. A recent meta-analysis of 10 studies conducted to estimate the diagnostic accuracy of LAMP for pulmonary tuberculosis revealed a sensitivity of $80.0 \%$ (95\% confidence interval (CI), $78 \%-83 \%)$ and a specificity of $96.0 \%(95 \% \mathrm{CI}$, 95.0\%-97.0\%) [9]. Furthermore, LAMP, which, in combination with PURE, has recently been established as a diagnostic technique requiring only limited equipment and manpower, provides an accessible, cost-effective, rapid, and more appropriate molecular diagnostic tool for tuberculosis in the field setting [6]. All procedures, including the time required for sample preparation, can be performed within just 1.5 hours.
FNA is an appropriate procedure to use in the initial evaluation in diagnostic methods targeting cervical nodular lesions, including TB lymphadenitis $[10,11]$. Although FNA is a non-invasive, inexpensive, viable alternative to excisional biopsy of the lymph nodes, the detection rate of $M$. tuberculosis by examining aspirate specimens is disappointingly low compared to microbiological techniques. Some researchers have suggested that a combination of FNA and PCR might increase the sensitivity for diagnosing TB lymphadenitis [12]. However, the overall sensitivity of PCR with aspirate specimens is significantly lower than that with biopsy specimens and histopathological examinations [13]. PCR is also notorious for yielding false-negative results. It is thus important to establish a rapid, highly sensitive molecular diagnostic method for use with FNA specimens before proceeding to more invasive procedures such as additional excisional biopsy of the lymph nodes. Although excisional biopsy for microbiological, molecular biological and histopathological evaluations has the highest diagnostic yield [11,14], it is also the most invasive means of reaching a diagnosis. LAMP, on the other hand, is a rapid diagnostic procedure that also plays an important role in deciding which specimen-collecting procedure to employ.

\section{Conclusions}

LAMP combined with PURE enabled us to detect $M$. tuberculosis from swabbed samples taken from an ulcerative lesion that had purulent exudate from the lymph nodes. The procedure could be performed in just 1.5 hours, making it beneficial and convenient for use in resource-limited, disease-endemic regions. We believe that the new commercial LAMP kit not only will be useful as a primary testing tool for TB but also will provide additional diagnostic value for TB lymphadenitis. Further studies are necessary to evaluate the sensitivity and specificity of this diagnostic tool.

\section{Consent}

The Ethics Committee of the National Defense Medical College Research approved the design and protocol of this study (reference no. 1205). Written informed consent was obtained from the patient for publication of this case report and any accompanying images. A copy of the written consent is available for review by the Editor-in Chief of this journal.

\section{Abbreviations}

FNA: Fine-needle aspiration; LAMP: Loop-mediated isothermal amplification; PCR: Polymerase chain reaction; PURE: Procedure for ultrarapid extraction; TB: Tuberculous.

\section{Competing interests}

The authors declare that they have no competing interests. 


\section{Authors' contributions}

Skaw conceived the study, participated in its design and coordination and helped draft the manuscript. TM was directly involved in the overall care of the patient and drafted the manuscript. JW, YF, KM, YH, SKan, FK, YM and AK were involved in data investigation and the patient's treatment. All authors read and approved the final manuscript.

\section{Acknowledgements}

We thank Dr Takashi Oda for helpful comments and suggestions.

\section{Author details}

${ }^{1}$ Division of Infectious Diseases and Pulmonary Medicine, Department of Internal Medicine, National Defense Medical College, 3-2, Namiki,

Tokorozawa, Saitama 359-8513, Japan. ²Division of Hematology, Department of Internal Medicine, National Defense Medical College, 3-2, Namiki, Tokorozawa, Saitama 359-8513, Japan. ${ }^{3}$ Department of Global Infectious Diseases and Tropical Medicine, National Defense Medical College, 3-2, Namiki, Tokorozawa, Saitama 359-8513, Japan.

Received: 16 March 2014 Accepted: 11 June 2014

Published: 16 July 2014

\section{References}

1. Geldmacher $H_{\text {, Taube }}$, Kroeger C, Magnussen H, Kirsten DK: Assessment of lymph node tuberculosis in northern Germany: a clinical review. Chest 2002, 121:1177-1182.

2. George G, Mony P, Kenneth J: Comparison of the efficacies of loopmediated isothermal amplification, fluorescence smear microscopy and culture for the diagnosis of tuberculosis. PLoS One 2011, 6:e21007.

3. Boehme CC, Nabeta P, Henostroza G, Raqib R, Rahim Z, Gerhardt M, Sanga E, Hoelscher M, Notomi T, Hase T, Perkins MD: Operational feasibility of using loop-mediated isothermal amplification for diagnosis of pulmonary tuberculosis in microscopy centers of developing countries. J Clin Microbio/ 2007, 45:1936-1940

4. Meier T, Eulenbruch HP, Wrighton-Smith P, Enders G, Regnath T: Sensitivity of a new commercial enzyme-linked immunospot assay (T SPOT-TB) for diagnosis of tuberculosis in clinical practice. Eur J Clin Microbiol Infect Dis 2005, 24:529-536.

5. Bloemberg GV, Voit A, Ritter C, Deggim V, Böttger EC: Evaluation of COBAS $^{\oplus}$ TaqMan $^{\circledast}$ MTB for direct detection of the Mycobacterium tuberculosis complex in comparison with Cobas Amplicor MTB. J Clin Microbiol 2013, 51:2112-2117.

6. Mitarai S, Okumura M, Toyota E, Yoshiyama T, Aono A, Sejimo A, Azuma Y, Sugahara K, Nagasawa T, Nagayama N, Yamane A, Yano R, Kokuto H, Morimoto K, Ueyama M, Kubota M, Yi R, Ogata H, Kudoh S, Mori T: Evaluation of a simple loop-mediated isothermal amplification test kit for the diagnosis of tuberculosis. Int J Tuberc Lung Dis 2011, 15:1211-1217.

7. Cohen RA, Muzaffar S, Schwartz D, Bashir S, Luke S, McGartland LP, Kaul K: Diagnosis of pulmonary tuberculosis using PCR assays on sputum collected within 24 hours of hospital admission. Am J Respir Crit Care Med 1998, 157:156-161

8. Geojith G, Dhanasekaran S, Chandran SP, Kenneth J: Efficacy of loop mediated isothermal amplification (LAMP) assay for the laboratory identification of Mycobacterium tuberculosis isolates in a resource limited setting. J Microbiol Methods 2011, 84:71-73.

9. Yuan LY, Li Y, Wang M, Ke ZQ, Xu WZ: Rapid and effective diagnosis of pulmonary tuberculosis with novel and sensitive loop-mediated isothermal amplification (LAMP) assay in clinical samples: a meta-analysis. J Infect Chemother 2014, 20:86-92.

10. Lau SK, Wei WI, Hsu C, Engzell UC: Efficacy of fine needle aspiration cytology in the diagnosis of tuberculous cervical lymphadenopathy. J Laryngol Otol 1990, 104:24-27.

11. Fontanilla JM, Barnes A, von Reyn CF: Current diagnosis and management of peripheral tuberculous lymphadenitis. Clin Infect Dis 2011, 53:555-562.

12. Singh KK, Muralidhar M, Kumar A, Chattopadhyaya TK, Kapila K, Singh MK, Sharma SK, Jain NK, Tyagi JS: Comparison of in house polymerase chain reaction with conventional techniques for the detection of
Mycobacterium tuberculosis DNA in granulomatous lymphadenopathy. J Clin Pathol 2000, 53:355-361.

13. Tachibana T, Orita Y, Fujisawa M, Nakada M, Ogawara Y, Matsuyama Y, Abe I, Sato Y, Uesaka K, Nishizaki K: Factors that make it difficult to diagnose cervical tuberculous lymphadenitis. J Infect Chemother 2013, 19:1015-1020.

14. Lee KC, Tami TA, Lalwani AK, Schecter G: Contemporary management of cervical tuberculosis. Laryngoscope 1992, 102:60-64.

doi:10.1186/1752-1947-8-254

Cite this article as: Kawano et al: Successful diagnosis of tuberculous lymphadenitis by loop-mediated isothermal amplification of cutaneous samples from an ulcerated surface lesion: a case report. Journal of Medical Case Reports 2014 8:254.

\section{Submit your next manuscript to BioMed Central and take full advantage of:}

- Convenient online submission

- Thorough peer review

- No space constraints or color figure charges

- Immediate publication on acceptance

- Inclusion in PubMed, CAS, Scopus and Google Scholar

- Research which is freely available for redistribution

Submit your manuscript at www.biomedcentral.com/submit
C Biomed Central 